\title{
THE SOCIOEMOTIONAL EFFECTS OF A COMPUTER-SIMULATED ANIMAL ON CHILDREN'S EMPATHY AND HUMANE ATTITUDES*
}

\author{
YUEH-FENG LILY TSAI \\ DAVID M. KAUFMAN \\ Simon Fraser University, Canada
}

\begin{abstract}
This study investigated the potential of using a computer-simulated animal in a handheld virtual pet videogame to improve children's empathy and humane attitudes. Also investigated was whether sex differences existed in children's development of empathy and humane attitudes resulting from play, as well as their feelings for a virtual pet. The results showed that after playing Nintendogs for 3 weeks, the participants of both sexes, on average, scored higher levels of empathy on the Bryant Empathy Index, and had higher levels of humane attitudes on the Intermediate Attitude Scale, compared to their pretest scores before they played. A statistical association also was revealed between time playing with a computer-simulated animal and improved scores in empathy and humane attitudes toward animals. The findings also showed that participants tended to form emotional attachments with their virtual pet and considered it a real pet.
\end{abstract}

\section{INTRODUCTION}

Research has shown that children's care of real pet animals is associated with higher levels of empathy and more positive attitudes toward the humane treatment of animals (Paul, 2000; Paul \& Sherpell, 1993; Vidovic, Stetic, \& Bratko, 1999). Studies have also shown that interacting with real pet animals can provide children with opportunities to practice and learn important social and emotional skills

\footnotetext{
*We gratefully acknowledge the support of the Social Sciences and Humanities Research Council of Canada (SSHRC) for providing funding through an INE Collaborative Research Initiative grant for the $S A G E$ for Learning project under which this work was completed.
} 
that include role taking, perspective taking, and language skills (Levinson, 1978; Myers, 1998; Nielsen \& Delude, 1989). Furthermore, children are provided with opportunities to experience different emotions (Melson, 2003; Myers, 1998; Nielsen \& Delude,1989; Rost \& Hartmann, 1994). The research has therefore suggested that owning a real pet animal may help promote children's socioemotional development including empathy, responsibility, and the care for others. The literature has also suggested that pets can be used as a sex-neutral medium that is effective in enhancing the nurturance behaviors of both girls and boys (Melson, 2003; Melson \& Fogel, 1989, 1996).

Although research has indicated that children can benefit socially and emotionally from interacting with pets, not all children have opportunities to interact with real animals due to possible reasons such as increased urbanization, allergies, as well as the expense and time of ownership. In recognition of the limitations but the desire of owning a pet, some entertainment software companies such as Bandai, Ubisoft, and Nintendo have created a pet replacement market. They have designed and promoted an artificial pet substitute, commonly referred to as a virtual pet, with computer-simulated characteristics similar to real pets. Some of these virtual pet products have become internationally popular. Bandai's Tamagotchi achieved record sales levels of over 40 million units worldwide since it was first released in 1997 (Bandai Corporation, 2008). Sony's AIBO, released in 1999, sold over 130,000 units in its first year (Sony Corporation, 2008). The more recent virtual pet product, Nintendogs, released in Japan on April 21, 2005 by Nintendo Japan, had first week sales of almost 200 thousand units (Jenkins, 2005). In Europe, about one million Nintendogs units were sold in the first 2 months (Nintendo Corporation, 2008), and in North America, almost a quarter million units were sold in the first week of release (Carless, 2005).

Most of the research studies that we reviewed investigated the effects of real pet animals on children's socioemotional development. Studies which examined the relationship between children's socioemotional development and their care of virtual pet animals were quite limited. With the growing popularity and sophistication of handheld mobile computer-based games such as Nintendogs, it now becomes increasingly more important to determine the socioemotional effects of computer game play involving a computer-simulated character. This includes whether children can develop empathy, as well as positive humane attitudes toward animals.

The purpose of this study was to investigate the potential of using a computersimulated animal in a handheld, commercially sold virtual pet videogame to improve children's empathy and their humane attitudes. The study also investigated whether there were any sex differences in children's development of empathy and attitudes resulting from playing and interacting with a virtual pet dog, as well as children's attitudes and feelings regarding a virtual pet dog. For this research, the game software Nintendogs was used. The Nintendogs game cartridges and Nintendo DS systems were bought entirely at retail cost without corporate sponsorship from Nintendo. Hopefully the understanding gained through this study about children's interaction with a computer-simulated animal 
may be applied to help develop new technology that can assist in the facilitation of children's social and emotional development.

\section{THEORETICAL FRAMEWORK}

\section{Empathy}

Empathy is considered an important element in child development, that is believed to influence children's prosocial development and altruistic behavior. Lack of empathy often resulted in aggressive and antisocial behavior (Davis, 1994). Therefore, encouraging children's development of empathy has been an important issue in children's education. Empathy is related to both cognitive and emotional development. Hoffman (1984) defined empathy as the "cognitive awareness of another person's internal states (thoughts, feelings, perceptions, intentions), and the vicarious affective response to another person" (p. 103). Feshbach (1978) further explicated that in addition to cognitive and emotional influences, children's social and life experiences interact to affect their empathic development. The importance of social and life experience therefore suggests that it may be possible to enhance children's empathy through proper guidance. Hoffman and Feshbach proposed that children's empathy could be enhanced through the provision of various life and emotional experiences, including through role-taking, perspective taking, and the greater use of language. They also supported opportunities for children to freely explore and express their emotions, and exercise their perception and understanding of emotions and non-emotional cues from social context. Furthermore, as theorized by German scholars such as Lipps, Vischer, and Lotze, the concept of aesthetic empathy suggests that non-human objects can stimulate empathic response (Gallese, 2003; Koss, 2006; Pigman, 1995; Verducci, 2000). Therefore, non-human objects may be able to enhance children's emotional and life experiences, as well as promote the development of their empathic abilities.

\section{Animal and Children's Development of Empathy and Humane Attitudes}

Numerous studies have suggested that animals may be able to enhance children's humane attitudes and empathic development, as well as abilities that relate to empathic development (Ascione, 1992, 2005; Ascione \& Weber, 1996; George, 1999; Levinson, 1972, 1978; Paul \& Serpell, 1993; Thompson \& Gullone, 2003 ). The positive influence of animals on empathic development and humane attitudes was also supported by Paul (2000), who concluded that there is "significant correlations between level and intensity of childhood pet relationships, concerns for the welfare of animals and empathy with humans" (p. 174). A review of the literature, which studied the influence of animals on children's enhancement of humane attitude and development of empathy, generally support the theory that interacting with animals can provide children 
with opportunities to practice role taking and perspective taking abilities. In order to communicate with animals, children also gained opportunities to practice language and learn how to perceive cues through interaction (Levinson, 1978; Myers, 1998). Through animals, Levinson suggested that children could be guided to progress away from egocentric thinking. This progression, according to Piaget's theories on cognitive development, is a necessary foundation for the development of empathy. Also through animals, children are provided with opportunities to experience different emotions, as well as to express freely their thoughts and emotions (Melson, 2003; Nielson \& Delude, 1989; Rost \& Hartmann, 1994). These cognitive and emotional abilities are important elements, which Hoffman and Feshbach considered are necessary for empathic development.

Furthermore, in animal and children's research, dogs are often considered as children's favorite animal playmates that have a strong effect. For example, Rost and Harmann (1994) compared children's interests in different types of pet animals, and concluded that most children prefer to own a dog. Kidd and Kidd (1985) also suggested that, compared to cats, children preferred dogs as their playmates. Daly and Morton (2006) further wrote that "children who own dogs would be more empathic than cat owners" (p. 122) and "... only children with dogs had significantly more positive attitude toward animals. There was no significance for those with cats, fish, birds, reptiles, or rodents" (p. 123).

\section{Sex Differences}

Studies have supported the hypothesis that females are more empathic than males (Feshbach, 1978; Feshbach \& Feshbach, 1969; Feshbach \& Roe, 1968; Kaluk, 1971; Powell, 1971 in Feshbach, 1978). However, whether girls are naturally more empathic than boys is a topic for continued research. Some researchers believe that socialization and social values may be possible reasons why females have been shown to be more empathic than males. Bryant (1982) found that younger children tend to show less sex differences in empathy, but by adolescence, girls show more empathy than boys do. In her conclusion, Bryant (1982) suggested that male teens might be more hesitant to share their feelings because of worries concerning social judgment. Feshbach and Roe (1968) also found that boys tend to be more inhibited and control their feelings. In thus doing, social constraint may be a reason that could have affected the empathy score results (Feshbach, 1978; Feshbach \& Roe, 1968). Eisenberg and Lennon (1983) assumed that the sex differences might be related to women's image and stereotype. They posited that "it is highly likely that females would be more willing than males to present themselves as being empathic and/or sympathetic, even if there were no real sex difference in responsiveness" (p. 125).

Research studies have also found that there are sex differences in the relationship between pet attachment and children's development of empathy. Girls usually showed stronger attachment to their pets and greater empathy than boys did (Rost \& Hartmann, 1994; Vidovic et al., 1999). Kidd and Kidd (1985) also 
concluded that boys reported less mutual love with pets compared to girls. However, Kidd and Kidd suggested that boys might have the same love toward pets as girls do, but due to sexual stereotyping and the pressure to conform, they were unwilling to report it. Although sex differences were found in pet attachment and empathy research, pets may still be considered as sex-neutral media for the development of caring behaviors. Nurturing and caring are two pro-social behaviors that relate to empathy. According to Melson and Fogel (1996) and Melson $(2001,2003)$ caring behaviors are often based on culture and sex. For example, women generally exhibit more caring and nurturing behaviors than males. The pattern usually starts from childhood where girls tend to take care of infant and younger siblings relatively more than boys do. However, Melson (2001) wrote that "caring for pets, unlike caring for babies, young children, or other people, is free of the sex-role association that typecast nurturance as an essentially feminine, perhaps quintessentially feminine, enterprise" (p. 55). Rost and Hartmann (1994) also found that although girls showed stronger desire in having a pet, boys were equally capable and willing to take care of a pet. Therefore, as a part of the theoretical framework of this research, it is assumed that pets could be used as a sex-neutral media to encourage empathy related caring behaviors.

\section{Virtual Pets and Children's Development of Empathy and Humane Attitudes}

If real animal pets can have potential benefits in enhancing children's empathic development and humane attitudes, would computer-simulated animals, also known as virtual pets, have the same effect? Although research on virtual pets is limited, there are some studies which indicated that virtual characters can influence children's emotional and empathic development. Research based on the anti-bullying software program, FearNot, found that the computer-simulated characters were able to evoke empathic responses such as distress and sympathy for the virtual victim character (Dias, Paiva, Vala, Aylett, Woods, Zoll, et al., 2006). Another study examined the impact of a research-designed robot called Sparky that simulated expressions when interacting with people, and found that Sparky's simulated expressions of sadness and fear were able to stir emotion among children (Scheeff, Pinto, Rahardja, Snibbe, \& Tow, 2002).

One research study about preschoolers' reasoning and interaction with a robot pet dog called AIBO found that, despite recognizing AIBO as a non-living robot, young children still believed that AIBO can feel pain (Kahn, Friedman, Perez-Granados, \& Freier, 2004). This belief discouraged them from treating the robot dog in ways they thought would be hurtful. The research also found that a majority of children believed that they could build a social relationship including the giving and receiving of mutual love from AIBO. Another study compared children's attitude and interaction toward a real pet dog versus that shown to AIBO (Melson, Kahn, Beck, Friedman, Roberts, \& Garret, 2005) and found that although children actually recognized AIBO is a robot dog, a majority 
of them believed that AIBO had emotional states as well as morality and the ability to socialize. Therefore, these limited studies on virtual pets lead to another aspect of the theoretical framework of this research that virtual characters may be able to evoke empathic response.

\section{RESEARCH QUESTIONS}

This research addressed the following questions:

1. Can playing and interacting with a virtual pet dog help promote children's development of empathy?

2. Can playing and interacting with a virtual pet dog help promote children's development of positive humane attitudes toward animals?

3. Are there sex differences in children's development of empathy and attitude resulting from playing and interacting with a virtual pet dog?

\section{RESEARCH METHODOLOGY}

\section{Sample}

The participants consisted of 51 children ( 26 boys and 25 girls) age 9 to 11 years in grades 4 or 5 from an elementary school in Surrey, a suburb of Vancouver, British Columbia. To voluntarily participate in the study with the permission of their parents, the children must not have played Nintendogs and do not own a real pet dog in order to limit past or present potential influences.

\section{Playing the Game}

Playing Nintendogs consists of caring, training, and competing with a computersimulated dog. There are several contests including disc competitions, agility trails, and obedience trails. Participants usually need to train their virtual pet dog before a prize can be won. The dog also has basic needs such as hunger and thirst, which players should meet by feeding and caring. Participants are able to interact with their virtual pet dogs by using a stylus to contact the touch screen, or by using a built-in microphone to speak to the dogs through the game's voice recognition system.

\section{Research Design}

This research study used a quasi-experimental repeated measures design with two pretests and a posttest as well as weekly logs and interview (see Figure 1).

\section{Procedures}

Fifteen participants, who formed the pilot, received one set of empathy and attitudes pretests prior to the experimental treatment of interacting with a virtual pet dog. This was done first in order to validate the instruments and experimental 
COMPUTER-SIMULATED ANIMALS AND CHILDREN / 109

\begin{tabular}{|l|l|l|l|l|l|}
\hline & O1 & & O2 & X & O3 \\
\hline Duration & & 3 weeks & & 3 weeks & \\
\hline Test & $\begin{array}{l}\text { Original } \\
\text { Measure }\end{array}$ & No intervnetion & $\begin{array}{l}\text { Pretest } \\
\text { Measure }\end{array}$ & Intervention & $\begin{array}{l}\text { Posttest } \\
\text { Measure }\end{array}$ \\
\hline $\begin{array}{l}\text { Number } \\
\text { of } \\
\text { Students }\end{array}$ & $n=36$ & & $\begin{array}{l}n=36 \\
\text { plus } \\
15 \text { (pilot) }\end{array}$ & & $\begin{array}{l}n=36 \\
\text { plus } \\
15 \text { (pilot) }\end{array}$ \\
\hline
\end{tabular}

Figure 1. Research design.

procedures. The other 36 participants were administered three repeated measures with two identical sets of pretests separated by 3 weeks as a control to determine any changes in children's empathy and humane attitude without the experimental treatment. The first set was termed "original" and the second set, "pretest." After the pretest, the participants were introduced to the features and functions of Nintendogs, and then loaned the game cartridge and Nintendo DS system for 3 weeks. Although the participants were asked to play with the game at least once each day if possible, they could decide the amount of time (i.e., duration) and how to play (i.e., interaction) with it. At the end of 3 weeks, the participants completed the empathy and attitudes posttests, and were interviewed regarding their feelings and ideas about interacting with their computer simulated virtual pet dog. The data from the pre and posttests, weekly logs, and interviews were used to examine the effect of interacting and taking care of a computer-simulated character on children's development of empathy as well as their attitudes concerning the humane treatment of animals.

\section{Instruments}

Two questionnaires were used in this study:

1. Bryant Index of Empathy for Children and Adolescents (Bryant 1982)The 22-item index was designed to assess human empathic tendencies in children, and has been used in other companion animal studies with children (Bryant, 1985; Malcarne, 1986, in Ascione, 1992). The reported coefficient alphas range from .54 to .79 (Bryant, 1982). The range of possible scores is from 0 to 22 with higher scores reflecting greater empathy; and

2. Intermediate Attitude Scale (Ascione, 1988)-The original scale was designed for use with 3rd, 4th, 5th, and 6th grade students in companion animal studies. It contains statements that a child can strongly agree, agree, disagree, or strongly disagree. The reported coefficient alpha is .70 , and the validity has been demonstrated (Ascione \& Weber, 1996). 
Since the original Intermediate Attitude Scale does not contain a neutral opinion selection, "undecided" was added in this study. Participants who chose more than one answer or did not choose any answer were included in this calculation. After the change, the range of possible scores is from 36 to 180 .

Both Bryant's Index and the Intermediate Attitude Scale were chosen for the research because they have been used in other relevant research studies, and were designed for the age group of the children in this study. An example question from the Bryant's Index: "It's silly to treat dogs and cats as though they have feelings like people." An example question from the Intermediate Attitude Scale: "A cat might feel lonely if it had no one to care for it over a weekend."

\section{FINDINGS}

\section{Reliability Coefficients}

The internal consistency reliability (Cronbach's Alpha) of the Bryant Empathy Index questionnaire for the multiple administrations ranged from .68 to .73 (see Table 1). The internal consistency reliability of the Intermediate Attitude Scale questionnaire for the multiple administrations ranged from .52 to .67 (see Table 1). The Companion Animal Bonding Revised Scale consisted of only the Posttest, and the Alpha coefficient ranged from .42 to.46.

\section{Empathy}

The Bryant Empathy Index (Bryant, 1982) was used for measuring the participants' level of empathy before and after the experimental intervention of the virtual pet dog in order to determine whether any changes in empathy score occurred after the treatment. The paired sample $t$ test of the Bryant Empathy Index-Original (BIorg) and the Pretest (BIpre) of the participant group $(n=36)$, prior to the experimental intervention, indicated that the mean score remained relatively stable (see Table 1$)$. The mean score for BIorg was $(M=13.78$, $S D=3.55)$ and for BIpre $(M=14.03, S D=3.61)$. The paired $t$-test was not statistically significant, $t(35)=.61, p=.54$. The test-retest reliability (Pearson Correlation Coefficient) between BIorg and BIpre was .77 $(n=36 ; p=.001)$.

After playing and interacting with the virtual pet for 3 weeks, paired sample statistical analysis showed that the posttest empathy score $(M=15.78, S D=3.56)$ on the Bryant Empathy Index (BIpst) for 51 participants increased an average of 1.86 points from the pretest score $(M=13.92, S D=3.66)$ on the 22-item questionnaire test (see Table 1). This result was statistically significant, $t(50)=$ $4.53, p=.001$. In the group of 36 participants who completed the questionnaire at all three points (original, pretest, posttest), the participants' empathy score increased an average of 2.33 points from the pretest score $(M=14.03, S D=3.61)$ on the same 22-item questionnaire (see Table 1). The result was statistically significant, $t(35)=5.30, p=.001$. 
Table 1. Paired Sample $t$-Test for Empathy Original vs. Pretest and Pretest vs. Posttest

\begin{tabular}{lccccc}
\hline & Scale & $n$ & Mean (SD) & $t$ & $p$ \\
\hline Pair 1 & Blorg & 36 & $13.78(3.55)$ & & \\
& Blpre & 36 & $14.03(3.61)$ & .61 & .54 \\
& & & & & \\
Pair 2 & Blpre & 36 & $14.03(3.61)$ & & \\
$\begin{array}{l}\text { (same } \\
\text { participants }\end{array}$ & Blpst & 36 & $16.36(3.18)$ & 5.30 & .001 \\
as pair 1) & & & & & \\
Pair 2 & & & & & \\
(all participants) & Blpre & 51 & $13.92(3.66)$ & & \\
\hline
\end{tabular}

\section{Humane Attitudes}

The Intermediate Attitude Scale (Ascione, 1988) was used to measure participants' humane attitude scores before and after the experimental intervention of a virtual pet dog in order to determine whether and what, if any, changes occurred after the treatment. The paired sample $t$-test of the Intermediate Attitude Scale-Original (IASorg) and the Pretest (IASpre) of the participant group $(n=36)$, prior to the experimental intervention, indicated that the mean score remained relatively stable (see Table 2). The mean score for IASorg was121.31 $(S D=11.60)$ and for IASpre was $121.36(S D=11.64)$. The paired $t$-test was not statistically significant, $t(35)=.04 ; p=.97$. The test-retest reliability (Pearson Correlation Coefficient) between IASorg and IASpre to was .70 $(n=36 ; p=.001)$.

After playing and interacting with the virtual pet dog for 3 weeks, paired sample statistical analysis showed that the posttest score $(M=128.57, S D=12.27)$ on the Intermediate Attitude Scale (IASpst) for 51 participants increased an average of 6.65 points from the pretest score $(M=121.92, S D=11.85)$ on the 36-items questionnaire test (see Table 2 ). The result was statistically significant, $t(50)=4.62, p=.001$. In the group of 36 participants who completed the questionnaire at all three points (original, pretest, posttest), the participants' empathy score increased an average of 8 points from the pretest score $(M=121.36$, $S D=11.64$ ) on the same 36 -items questionnaire (see Table 2). The result was statistically significant, $t(35)=4.39, p=.001$.

\section{Correlation between Empathy and Humane Attitude}

To investigate whether there were any statistically significant associations among participants' empathy and humane attitude, correlations between participants' Bryant's Empathy Index score (original, pretest, and posttest) and 
Table 2. Paired Sample $t$-Test for Humane Attitude Original vs. Pretest and Pretest vs. Posttest

\begin{tabular}{lccccc}
\hline & Scale & $n$ & Mean (SD) & $t$ & $p$ \\
\hline Pair 1 & IASorg & 36 & $121.31(11.60)$ & & \\
& IASpre & 36 & $121.36(11.64)$ & .04 & .97 \\
& & & & & \\
Pair 2 & IASpre & 36 & $121.36(11.63)$ & & \\
(same & IASpst & 36 & $129.36(12.33)$ & 4.39 & .00 \\
participants & & & & & \\
as pair 1) & & & & & \\
& & & & & \\
Pair 2 & IASpre & 51 & $121.92(11.85)$ & & \\
(all participants) & IASpst & 51 & $128.57(12.27)$ & 4.62 & .00 \\
\hline
\end{tabular}

Intermediate Attitude Scale score (original, pretest, and posttest ) were calculated and analyzed.

The correlation between participants' original scores in empathy (BIorg) and humane attitude (IASorg) were positive and significant $(r=.36, p=.03$ ). Using Cohen's (1988) guidelines, this effect size is small. The correlation between participants' pretests in empathy (BIpre) and humane attitude (IASpre) were positive and significant $(r=.56, p=.00)$. Using Cohen's (1988) guidelines, this effect size is medium. The correlation between participants' posttests in empathy (BIpst) and humane attitude (IASpst) were positive and significant $(r=.48$, $p=.00)$. Using Cohen's (1988) guidelines, this effect size is small. Therefore, the correlation results indicated that participants who had higher empathy scores tended to have higher humane attitude scores in the original test, pretest, and posttest.

\section{Sex Differences in Children's Development of Empathy and Humane Attitudes}

Twenty-five female and 26 male children participated in the research. This included eight girls and seven boys in the pilot study group who only completed the pretest and posttest. Consequently, in total, there were 17 female and 19 male participants who completed the original tests, and 25 female and 26 male participants who completed the pretest and posttest.

\section{Bryant Empathy Index}

Table 3 shows that scores of female participants were significantly different from male participants on the Bryant Empathy Index on all three tests: original, BIorg, $t(35)=2.87, p=.01$; pretest, BIpre, $t(50)=3.06, p=.00$; and posttest, BIpst, $t(50)=2.80, p=.01$. However, the differences between females and 
Table 3. Comparison of Males and Females on Bryant Empathy Index Original/Pretest/Posttest

\begin{tabular}{llllll}
\hline & Sex & $n$ & Mean $(S D)$ & $t$ & $p$ \\
\hline Blorg & Female & 17 & $15.41(2.74)$ & 2.87 & .01 \\
& Male & 19 & $12.32(3.61)$ & & \\
Blpre & Female & 25 & $15.40(2.89)$ & 3.06 & .00 \\
& Male & 26 & $12.50(3.81)$ & & \\
\multirow{3}{*}{ Blpst } & Female & 25 & $17.12(2.33)$ & 2.80 & .01 \\
& Male & 26 & $14.50(4.07)$ & & \\
\hline
\end{tabular}

Table 4. Comparison of Males and Females on Humane Attitude Test Original/Pretest/Posttest

\begin{tabular}{lllccc}
\hline & Sex & $n$ & Mean $(S D)$ & $t$ & $p$ \\
\hline IASorg & Female & 17 & $124.53(12.40)$ & 1.61 & .12 \\
& Male & 19 & $118.42(10.31)$ & & \\
IASpre & Female & 25 & $124.64(11.97)$ & 1.63 & .11 \\
& Male & 26 & $119.31(11.35)$ & & \\
\multirow{3}{*}{ IASpst } & Female & 25 & $130.04(11.81)$ & .84 & .41 \\
& Male & 26 & $127.15(12.77)$ & & \\
\hline
\end{tabular}

males on the Intermediate Attitude Scale on all three tests were not significant: original, IASorg, $t(35)=1.61, p=.12$; pretest, IASpre, $t(50)=1.63, p=.11$; and posttest, IASpst, $t(50)=.84, p=.41$ (see Table 4).

A review of the original test, pretest, and posttest of male and female participant mean scores indicated that the Bryant Empathy Index mean scores for female participants: BIorg $(n=17)$ was 15.41, $(S D=2.74)$; BIpre $(n=25)$ was15.40, $(S D=2.89)$; and BIpst $(n=25)$ was $17.12,(S D=2.33)$ (see Table 3$)$. These scores were higher than the mean scores for male participants: BIorg $(n=19, M=12.32, S D=3.61)$; BIpre $(n=26, M=12.50, S D=3.81)$; BIpst $(n=26, M=14.50, S D=4.07)$ (see Table 3$)$.

Therefore, the Bryant Empathy test showed that female participants had a slightly higher mean score than the mean score of male participants before and after the experimental intervention of the virtual pet dog. However, both female and male participants showed stable scores before the introduction of the virtual dog intervention: Female: $n=17$; BIorg, $M=15.41, S D=2.74$; BIpre, $M=16.24$, $S D=2.33, t(16)=1.36, p=.19$; Male: $n=19$; BIorg, $M=12.32, S D=3.61$; BIpre, 
$M=12.05, S D=3.42, t(18)=.49, p=.63$. Both female and male participants showed relatively higher mean scores after the 3-week experimental intervention of the virtual dog: Female: $n=25$; BIpre, $M=15.40, S D=2.89$; BIpst, $M=17.12$, $S D=2.33, t(24)=3.13, p=.01$; Male, $n=26$; BIpre, $M=12.50, S D=3.81$; BIpst, $M=14.50, S D=4.07, t(25)=3.23, p=.00$.

\section{Intermediate Attitude Scale}

A review of the original test, pretest, and posttest of male and female participant mean scores indicated that the Intermediate Attitude Scale mean scores for female participants in: IASorg $(n=17)$ was $124.53,(S D=12.40)$; IASpre $(n=25)$ was $124.64,(S D=11.97)$; IASpst $(n=25)$ was $130.04,(S D=11.81)$ (see Table 4). These scores were higher than the mean scores for male participants: IASorg $(n=19, M=118.42, S D=10.31)$; IASpre $(n=26, M=119.31$, $S D=11.35)$; and IASpst $(n=26, M=127.15, S D=12.77)$ (see Table 4). Therefore, analysis using the humane attitude scale showed that female participants had a slightly higher mean score than male participants before and after the experimental intervention of the virtual pet dog. However, both female and male participants showed stable scores before the introduction of the virtual dog intervention: Female: $n=17$; IASorg $M=124.52, S D=12.40$; IASpre $M=127.24$, $S D=10.07, t(16)=1.20, p=.27$; Male: $n=19$; IASorg $M=118.42, S D=10.31$; IASpre $M=116.11, S D=10.56, t(18)=1.24, p=.23$. Also, both female and male participants showed relatively higher mean scores after the 3-week experimental intervention of the virtual dog: Female: $n=25$; IASpre $M=124.64, S D=11.97$; IASpst $M=130.04, S D=11.81, t(24)=3.09, p=.01$; Male: $n=26$; IASpre $M=119.31, S D=11.35$; IASpst $M=127.15, S D=12.77, t(25)=3.44, p=.001$.

\section{Activity Differences}

The sexes were compared in terms of play duration, incidents of caring behaviors, incidents of training behaviors and incidents competing using data collected through the participants' self-recorded weekly log. The results showed that there were no significant differences in all three variables: play duration, $t(50)=1.51, p=.14$; incidents of caring behaviors, $t(50)=1.39, p=$ .17 ; incidents of training behaviors, $t(50)=.10, p=.92$; incidents competing, $t(50)=1.33, p=.19($ see Table 5$)$.

\section{Children's Perception of a Virtual Pet Dog}

During the post-experimental treatment interview, participants were asked the question: "Do you think the Nintendog is a pet or a video game and why?" A majority of the participants $(92.2 \%)$ answered that their virtual dog is a pet rather than a video game. Only $3.9 \%$ of the participants believed it was a video game, and $3.9 \%$ of the participants believed it was both a video game and a pet. For participants who suggested that the virtual dog was a real pet, most (52.4\%) asserted that it looked and acted real. A third (33.3\%) also mentioned 
COMPUTER-SIMULATED ANIMALS AND CHILDREN ／ 115

Table 5. Comparison of Male and Female Activity Differences

\begin{tabular}{lllccc}
\hline & Sex & $n$ & Mean $(S D)$ & $t$ & $p$ \\
\hline Play duration & $\begin{array}{l}\text { Female } \\
\text { Male }\end{array}$ & 25 & $1328.96(813.25)$ & 1.51 & .14 \\
& & 26 & $1022.58(629.35)$ & & \\
Incidents of caring & Female & 25 & $288.56(123.84)$ & 1.39 & .17 \\
behaviors & Male & 26 & $360.54(299.55)$ & & \\
Incidents of training & Female & 25 & $142.08(100.04)$ & .10 & .92 \\
behaviors & Male & 26 & $144.73(93.22)$ & & \\
Incidents of & Female & 25 & $52.84(12.51)$ & 1.33 & .19 \\
competing & Male & 26 & $46.23(17.09)$ & & \\
\hline
\end{tabular}

that virtual dogs need care just as do real dogs. For example, one child expressed the needs of the virtual dog: "It's just like taking care of a real dog. You got to feed it, you got to give it water, you got to take it for a walk, you got to clean up after it. You got to enter it into competitions, just like taking care of a real dog." Three participants (4.8\%) indicated that the virtual dog has feelings. For example, one child said, "I think it's a pet because it's like she has feelings." Three participants considered their virtual dog to be real and used it as a substitute for a real dog. One child said, "A pet, because it is a dog. I want to get a dog and I am too young for it. I need too wait until 11 and I will get a dog."

\section{DISCUSSION}

\section{Virtual Pets and Children's Development of Empathy}

Animal research studies have suggested that animals could play effectual roles in enhancing children's empathic development or abilities that relate to empathic development (Ascione, 1992, 2005; Ascione \& Weber, 1996; George, 1999; Levinson, 1972, 1978; Myers, 1998). Research studies on virtual characters have suggested that virtual characters may be able to evoke empathic responses by children (Dias et al., 2006; Scheef et al., 2002). However, studies have not been identified that directly found a conclusive association that playing, interacting with, and responding to a virtual pet dog could promote children's development of empathy.

In this research, the findings support the assumption that a virtual pet dog could be used as a medium to facilitate the development of children's empathy. The study found that the participants' scores on the Bryant Empathy Index remained fairly stable during the first 3 weeks without the experimental intervention of a virtual pet dog, real pet dog, or the provision of any explicit lessons 
on teaching empathy to children ( $n=36$; BIorg, $M=13.78, S D=3.55$; BIpre, $M=14.03, S D=3.61, t(35)=.61, p=.54$.). However, after playing and interacting with a virtual pet dog for 3 weeks, it was found that the participants' scores on the Bryant Empathy Index had increased statistically significantly $(n=51$, BIpre, $M=13.92, S D=3.66$; BIpst, $M=15.78, S D=3.56, t(50)=4.53, p=.001)$; ( $n=36$; BIpre, $M=14.03, S D=3.61$; BIpst, $M=16.36, S D=3.18, t(35)=5.30$, $p=.001)$. Therefore, the findings seem to suggest and support the hypothesis that a virtual pet dog may be able to help enhance children's empathic development.

\section{Virtual Pets and Children's Enhancement of Humane Attitudes}

Research studies have suggested that direct interaction with animal pets or farm animals could enhance children's humane attitudes (George, 1999). The research from Paul and Serpell (1993) supported George's theory and further elaborated that the experience of pet ownership during childhood may help children to develop positive humane attitudes toward animals and people. However, studies have not been found which conclusively established an association between interacting with a virtual pet and the enhancement of humane attitude.

In this research, the findings support the assumption that a virtual pet dog could be used as a medium to facilitate the enhancement of children's humane attitudes. The study found that participants' scores in the Intermediate Attitude Scale remained fairly stable during the first 3 weeks without the experimental intervention of a virtual pet dog, real pet dog, or the provision of any explicit lessons on teaching empathy to children $(n=36$; IASorg, $M=121.31, S D=11.60$; IASpre, $M=121.36, S D=11.64, t(35)=.04 ; p=.97)$. However, after playing and interacting with a virtual pet dog for 3 weeks, it was found that the participants' scores on Intermediate Attitude Scales had improved ( $n=51$; IASpre, $M=121.92, S D=11.85$; IASpst, $M=128.57, S D=12.27, t(50)=4.62, p=.001)$; ( $n=36$, IASpre, $M=121.36, S D=11.64$; IASpst, $M=129.36, S D=12.33$, $t(35)=4.39, p=.001)$. Therefore, the findings of this research suggest and support the hypothesis that a virtual pet dog may be able to enhance children's humane attitudes toward animals through playing and taking care of a virtual pet dog.

\section{Sex Differences}

The research found that female participants tended to have higher empathy test scores than male participants before and after the experimental intervention with the virtual pet dog (Female: BIorg, $n=17, M=15.41, S D=2.74$; BIpre, $n=25, M=15.40, S D=2.89$; BIpst, $n=25, M=17.12, S D=2.33$; Male: BIorg, $n=19, M=12.32, S D=3.61$; BIpre, $n=26, M=12.50, S D=3.81$; BIpst, $n=26$, $M=14.50, S D=4.07)$, and that the differences in scores are statistically significant $(\mathrm{BIorg}, t(35)=2.87, p=.01$; BIpre, $t(50)=3.06, p=.00$; BIpst, $t(50)=2.80$, $p=.01)$. Furthermore, the research found that both females and males' empathy posttest scores improved after the 3-week experimental intervention of the virtual pet dog. Analysis based on the Intermediate Attitude Scale questionnaire 
found that female participants had higher humane scores than males (Female: IASorg, $n=17, M=124.53, S D=12.40$; IASpre, $n=25, M=124.64$, $S D=11.97$; IASpst , $n=25, M=130.04, S D=11.81$; Male: IASorg, $n=19, M=118.42$, $S D=10.31$; IASpre, $n=26, M=119.31, S D=11.35$; IASpst, $n=26, M=127.15$, $S D=12.77$ ). However, the differences were not statistically significant (IASorg, $t(35)=1.61, p=.12$; IASpre, $t(50)=1.63, p=.11$; IASpst, $t(50)=.84, p=.41)$. Both female and male participants' humane attitude scores improved after the 3 -week experimental intervention with a virtual pet dog (Female: $n=25$; IASpre $M=124.64, S D=11.97$; IASpst $M=130.04, S D=11.81, t(24)=3.09, p=.01$; Male: $n=26$; IASpre $M=119.31, S D=11.35$; IASpst, $M=127.15, S D=12.77$, $t(25)=3.44, p=.001)$. Further analysis that compared the duration and types of activities (e.g., caring, training, and competing) did not reveal any statistically significant difference between female and male participants (play duration, $t(50)=1.51, p=.14$; incidents of caring behaviors $t(50)=1.39, p=.17$; incidents of training behaviors, $t(50)=.10, p=.92$; incidents competing, $t(50)=1.33$, $p=.19$ ).

The findings of this research support the conclusion of the empathy study by Vidovic, Stetic, and Bratko (1999) which concluded that girls are more inclined to be attached to animals, and are also more empathic than boys. The findings also support Rost and Hartman (1994), which suggested that boys and girls are equally capable and willing to take care of pets, because no significant difference was found between female and male participants regarding the duration of interaction and the type of activities that the participants engaged in, including caring, training, and competing behaviors. Consequently, despite girls being found to be more empathic than boys, animals could be used as a sex-neutral media to encourage empathy-related caring behaviors (Melson, 2003). In summary, both female and male participants had improvements in empathy and humane attitude posttest scores after the 3-week experimental intervention of playing and taking care of a virtual pet dog. No sex differences were found regarding the types of activities that the participants engaged in, nor in the time devoted to playing with virtual pet dogs. Therefore, this research suggests that virtual pet dogs could also be used as a sex-neutral medium to enhance children's development of empathy and humane attitudes.

\section{Attitudes and Feelings Regarding a Virtual Pet Dog}

After playing with, interacting with, and responding to the virtual pet dogs, participants received a semi-structured interview in which they were encouraged to express their feelings and attitudes toward their virtual pet dog. The research found that a majority of the participants $(92.16 \%)$ considered their virtual pet to be a real pet rather than a video game. Half of the participants $(52.38 \%)$ said that their virtual pet was a real pet instead of a video game because their dog appeared or behaved like a real dog. A third of the participants (33.33\%) said that their virtual pet was like a real pet because it had physical needs and required care. These findings raise a new set of interesting and important questions. 


\section{Limitations and Suggestions for Future Research}

The analysis of the quantitative data from the measurement instruments and the qualitative data from the semi-structure interviews resulted in many findings. There were also some questions that remained unanswered as well as ideas that need further study. Some possible ways in which the research-design could be improved in future studies:

1. Expand the number of research group participants: The study used a quasiexperimental repeated measurement. Because of the nature of the study, many schools that were approached about hosting declined, citing that the administrators or teachers did not personally like the idea of children using a video game in school even for research purposes. It would be ideal if the research can use a sample which better reflects the total students in Vancouver urban areas. Hopefully, the results of this research can encourage more schools to participate in related computer research in the future.

2. Provide a longer experimental duration: This research was designed to have an experimental intervention period of 3 weeks to avoid excessively disrupting the school's curriculum and students' routines. Future research should be designed with an extended research period in order to determine whether a virtual pet dog could have long term effect on children's attitude and empathic development.

3. Use or create additional reliable instruments for the virtual pet research: The data of participants' play duration and interaction activities were based on the participants' self-recorded weekly log. It may have been subjected to recording errors due to under- or over-estimating the amount of time that they actually played and how they played with the virtual pet. To record accurately the play and interaction data, future research might need to be done under clinical conditions using video recording. Also other measurement instruments could be created.

\section{CONCLUSION}

Children's empathic development can be influenced by many different factors related to cognitive, emotional, and life experiences (Feshbach, 1978, 1979, 1982; Hoffman, 1982, 1987, 2000). The possibility that animals may have the potential to contribute to children's development of empathy has been suggested by research including Levinson (1972, 1978), George (1999), Myers (1998), and Paul (2000). Other research (Dias et al., 2006; Scheeff et al., 2002) have found that virtual characters can elicit emotional and empathic responses from children. In this study, which used the handheld computer game Nintendogs, the researchers attempted to answer the question whether a computer-simulated animal can enhance children's empathic and humane attitude development. The results found that, after playing Nintendogs for 3 weeks, male and female participants on average scored higher scores of empathy on the Bryant Empathy Index and higher scores of humane attitude on the Intermediate Attitude Scale, compared to their 
pretest scores before they played with the virtual pet. In thus doing, a statistical association was revealed between playing a computer-simulated animal with improved scores in empathy and humane attitude toward animals. In addition, the research suggested that a virtual pet dog may be used as a gender-neutral media to enhance children's development of empathy and humane attitudes. This research further showed that the participating children tended to regard the virtual pet dog as a real dog instead of a toy or game, and treated it with care.

In this research study, the findings regarding the potential of whether playing with a computer-simulated animal can enhance children's empathy and humane attitude toward animals were reported, along with sex differences and attitudes toward the virtual pet dog. More findings will be reported in follow-up papers, which discuss how factors such as duration and type of interaction with the computer-simulated character, as well as the detailed interview reported of children's feelings and experiences in interacting with a virtual pet dog. Due to practical research difficulties, this study used repeated measures as the research design. If possible, the researchers suggest future studies should enlarge the number of participants and use an independent control group. Although this research has initially showed a positive relationship between playing with a virtual pet and higher empathic and humane attitude scores, it is important to conduct further studies that involve a longer duration with more participants to explore the long term influence of whether a computer-simulated character can make a difference in the enhancement of children's empathic development and humane attitudes.

In conclusion, although the research would not suggest that virtual pets could replace real pets, the study did show that most children had positive interactional experiences with their virtual pet dogs. The research further found that playing and taking care of a virtual pet were positively correlated with the participants' higher empathic and humane attitude posttest scores. In thus doing, the research suggests that playing and caring for a virtual pet may be able to enhance children's development of empathy and humane attitudes, and thereby serves to support future research into the effects of virtual pets on children's development. Furthermore, computer games such as Nintendogs could possibly be used as a component of an educational unit to address curricular goals regarding the promotion of social responsibility in schools. The educational unit could incorporate a multimedia approach comprising elements such as print, video, and even computer games with simulated virtual characters to help facilitate the social and emotional development of children.

\section{REFERENCES}

Ascione, F. R. (1988) Intermediate Attitude Scale. Assessment of third through sixth graders' attitude toward the treatment of animals. Logan, UT: Wasatch Institute for Research Evaluation.

Ascione, F. R. (1992). Enhancing children's attitudes about the humane treatment of animals: Generalization to human-directly empathy. Anthrozoo, 5(3), 176-191. 
Ascione, F. R. (2005). Children and animals: Exploring the roots of kindness and cruelty. West Lafayette, IN: Purdue University Press.

Ascione, F. R., \& Weber, C. V. (1996). Children's attitudes about the humane treatment of animals empathy: One year follow up of a school based intervention. Anthrozoo, 9(4), 188-195.

Bandai Corporation. (2008). Ultimate Tamagotchi family reunion makes more family more fun. (Bandai Press Release April 2, 2008). Retrieved May 8, 2008 from: http://www.bandai.com/about/pr.cfm?pr_id=174

Bryant, B. K. (1982). An index of empathy for children and adolescents. Child Development, 53(2), 413-425.

Bryant, B. K. (1985). The neighborhood walk: Sources of support in middle childhood. Monographs of the Society for Research in Child Development, serial no. 210, 50(3). Chinchaga, IL: University of Illinois Press.

Carless, S. (2005, September 1). Nintendo reveals impressive U.S. Nintendogs figures. Message posted to Gamasutra Industry News. Retrieved May 10, 2008, from http://gamasutra.com/php-bin/news_index.php?s

Cohen, J. (1988). Statistical power and analysis for the behavioral sciences (2nd ed.). Hillsdale, NJ: Lawrence Erlbaum Associates.

Daly, B., \& Morton, L. L. (2006). An investigation of human-animal interactions and empathy as related to pet preference, ownership, attachment, and attitudes in children. Anthrozoos, 19(2), 113-127.

Davis, M. H. (1994). Empathy: A social psychological approach. Dubuque, IA: Wm. C. Brown.

Dias, J., Paiva, A., Vala, M., Aylett, R., Woods, S., Zoll, C., et al. (2006). Empathic characters in commuter-based personal and social education. In M. Pivec (Ed.), Affective and emotional aspects of human-computer interaction (pp. 246-254). Amsterdam, Netherlands: IOS Press.

Eisenberg, N., \& Lennon, R. (1983). Sex differences in empathy and related capacities. Psychology Bulletin, 94(1), 100-131.

Feshbach, N. D. (1978). Studies of empathic behavior in children. In B. A. Maher (Ed.), Progress in experimental personality research (Vol. 8, pp. 1-48). New York: Academic Press.

Feshbach, N. D. (1979). Empathy and training: A field study in affective education. In S. Feshbach \& A. Fraczek (Eds.), Aggression and behavior change (pp. 234-249). New York: Praeger.

Feshbach, N. D. (1982). Sex differences in empathy. In N. Eisenberg (Ed.), The development of prosocial behavior (pp. 315-338). New York: Academic Press.

Feshbach, N., \& Feshbach, S. (1969). The relationship between empathy and aggression in two groups. Developmental Psychology, 1, 102-107.

Feshbach, N. D., \& Roe, K. (1968). Empathy in six and seven year olds. Child Development, 39(1), 133-145.

Gallese, V. (2003). The roots of empathy: The shared manifold hypothesis and the neural basis of intersubjectivity. Psychopathology, 36, 171-180.

George, M. G. (1999). The role of animals in the emotional and moral development of children. In F. R. Ascione \& P. Arkow (Eds.), Child abuse, domestic violence, and animal abuse (pp. 380-392). West Lafayette, IN: Purdue University Press.

Hoffman, M. L. (1982). The development of prosocial motivation: Empathy and guilt. In N. Eisenberg (Ed.), The development of prosocial behavior (pp. 281-314). New York: Academic Press. 
Hoffman, M. L. (1984). Interaction of affect and cognition in empathy. In C. E. Izard, J. Kagan, \& R. B. Zajonc (Eds.), Emotions, cognition, and behavior (pp. 103-131). Cambridge, UK: Cambridge University Press.

Hoffman, M. L. (1987). The contribution of empathy to justice and moral judgment. In N. Eisenberg \& J. Strayer (Eds.), Empathy and its development (pp. 47-80). New York: Cambridge University Press.

Hoffman, M. L. (2000). Empathy and moral development. Cambridge, UK: Cambridge University Press.

Jenkins, D. (2005, April 28). Japanese scale charts, week ending April 24th. Message posted to Gamasutra Industry News. Retrieved May 10, 2008 from: http://gamasutra. com/php-bin/news_index.php?story $=5386$

Kahn, P. H., Friedman, B., Perez-Granados, D. R., \& Freier, N. G. (2004). Robotic pets in the lives of preschool children. Interaction Studies, 7(3), 405-436.

Kaulk, M. T. (1971). Empathy in five and six year olds. Unpublished master's thesis, Women's College of Duke University.

Kidd, A. H., \& Kidd, R. M. (1985). Children's attitudes toward their pets. Psychological Reports, 57, 15-31.

Koss, J. (2006). On the limits of empathy. Art Bulletin, 83(1), 139-157.

Levinson, B. M. (1972). Pets and human development. Springfield, IL: Charles C Thomas.

Levinson, B. M. (1978). Pets and personality development. Psychological Reports, 42, 1031-1038.

Melson, G. F. (2001). Why the wild things are: Animals in the lives of children. Cambridge, MA: Harvard University Press.

Melson, G. F. (2003). Child development and the human-companion animal bond. American Behavioral Scientist, 47(1), 31-39.

Melson, G. F., \& Fogel, A. (1989). Children's ideas about animal young and their care: A reassessment of gender differences in the development of nurturance. Anthrozoos, 2(4), 265-273.

Melson, G. F., \& Fogel, A. (1996). Parental receptions of their children's involvement with household pets. Anthrozoos, 9(2-3), 95-106.

Melson, G. F., Kahn, P. H., Beck, A. M., Friedman, B., Roberts, T., \& Garret, E. (2005). Robots as dogs? Children's interactions with the robotic dog AIBO and a live Australian shepherd. Paper presented at conference for Human-Computer Interaction. Retrieved July 17, 2007 from: http://faculty.washington.edu/pkahn/articles/Robots as_Dogs.pdf

Myers, G. (1998). Children and animals: Social development and our connections to other species. Boulder, CO: Westview Press.

Nielsen, J. A., \& Delude, L. A. (1989). Behavior of young children in the presence of different kinds of animals. Anthrozoos, 3(2), 119-129.

Nintendo Corporation. (2008). Europe loves Nintendogs. (Nintendo Press Release, 2005, November 28). Retrieved May 10, from http://nintendoworldreport.com/newsArt. cfm? artid $=10961$

Paul, E. S. (2000). Love of pets and love of people. In A. L. Podeberscek, E. S. Paul, \& J. A. Serpell (Eds.). Companion animals and us: Exploring the relationships between people and pets (pp. 168-186). Cambridge, UK: Cambridge University Press.

Paul, E., \& Serpell, J. (1993). Childhood pet keeping and humane attitudes in young adulthood. Animal Welfare, 2, 321-337.

Pigman, G. W. (1995). Freud and the history of empathy. International Journal of Psycho-Analysis, 76, 237-256. 
Powell, A. B. (1971). The nature of the empathic response in four, six, and ten year olds. Unpublished master thesis, University of California, Los Angeles.

Rost, D. H., \& Hartmann, A. (1994). Children and their pets. Anthrozoos, 7(4), 242-254.

Scheeff, M., Pinto, J., Rahardja, K., Snibbe, S., \& Tow, R. (2002). Experiences with Sparky, a social robot. In K. Dautenhahn, A. H. Bond, L. Canamero, \& B. Edmonds (Eds.), Socially intelligent agents: Creating relationships with computers and robots (pp. 173-180). Norwell, MA: Kluwer Academic Publishers.

Sony Corporation. (2008). Sony launches four-legged entertainment robot. (Sony Press Release 1999, May 11). Retrieved May 10 from: http://www.sony.net/SonyInfo/ News/Press_Archive/199905/99-046/index.html

Thompson, K. L., \& Gullone, E. (2003). Promotion of empathy and prosocial behavior in children through humane education. Australian Psychologist, 38(3), 175-182.

Verducci, S. (2000). A conceptual history of empathy and a question it raises for moral education. Educational Theory, 50(1), 63-80.

Vidovic, V. V., Stetic, V. V., \& Bratko, D. (1999). Pet ownership, type of pet and socioemotional development of school children. Anthrozoos, 12(4), 211-217.

Direct reprint requests to:

Dr. David M. Kaufman

Professor, Faculty of Education

Simon Fraser University

8888 University Drive

Burnaby, BC, Canada V5A 1S6

e-mail: dkaufman@sfu.ca 